\title{
SWIMMING OF SLENDER FISH IN A NON-UNIFORM VELOCITY FIELD
}

\author{
J. N. NEWMAN
}

(Received 28 January, 1975)

\begin{abstract}
The hydrodynamic pressure forces acting upon a slender fish are derived for the case of a fish swimming in a non-uniform velocity field. Possible applications are the effects on fish propulsion of swimming in waves, in turbulent eddies, and in the presence of other fish or a moving ship. The fish is assumed to be a slender body, with no vorticity shed into the fluid except at a single abrupt trailing edge located at the posterior end of the fish, and to be performing small lateral swimming undulations of its body. The non-uniform field through which the fish swims is assumed to be irrotational, and this field as well as the body undulations must be slowly-varying on the length-scale of the lateral fish dimensions. Expressions are derived for the local force and the time-averaged total thrust force. These are applied to the study of steady-state bow-riding and wave-riding of porpoises.
\end{abstract}

\section{Introduction}

The application of slender-body theory to fish swimming was initiated by Lighthill [5], and this theory has subsequently been extended by various authors. (For a recent survey see Newman and Wu, [8].) In his original analysis Lighthill [5] assumed that the fish body was slender, with an abrupt sharp trailing edge at the tail from which a vortex sheet extends downstream into the fluid, but with no vorticity shed into the fluid upstream of the tail. The fluid motion was assumed ideal, and lateral swimming undulations of the fish body were assumed small in amplitude and slowly-varying along the body axis. In the subsequent papers on this subject these assumptions have been critically examined and in some cases removed, but it has generally been assumed that the fish swims in a uniform infinite fluid which is at rest except for the perturbation field generated by the fish.

Interest in fish swimming in non-uniform fields is stimulated by considerations of the effects, on the swimming performance, of various external disturbances including the presence of other fish (fish-schooling), waves on the ocean surface, ambient turbulence (particularly in fast-flowing rivers), and the effects of a moving ship. In a particular example of the latter, which we shall 
examine here, porpoises are known to "bow-ride" near ships' bows, and various qualitative explanations for this phenomenum are noted by Lang [4]. Two-dimensional theories for swimming in non-uniform fields have been developed, especially for the case of ambient surface waves, in an analogous manner to the gust-theory of two-dimensional unsteady thin wing theory in aerodynamics. In addition Weihs [12] has studied fish schooling interactions using a steady two-dimensional approach. Since fish are typically slender rather than two-dimensional, it would seem desirable to develop the complementary theory, initiated by Lighthill [5] for a uniform field, and to extend this to the case where the surrounding fluid is not at rest but moving with some arbitrary non-uniform velocity field. In a recent paper Coene [2] has developed the slender-body approach for a fish swimming in waves. That work can be regarded as a special case of the present problem, but Coene's analysis is carried further to include detailed calculations of propulsive efficiency in waves.

In this paper the slender-body theory of fish propulsion is extended to the case where the surrounding fluid is in a state of non-uniform motion. In order to make our results applicable to analyses of the different external disturbances noted above, we shall suppose that the non-uniformity is quite general, it being necessary to assume only that this field is irrotational, with gradients which are small based on the lateral (i.e., slender) length scale of the fish. To keep the theory as simple as possible we make the same assumptions as Lighthill [5] regarding the fish slenderness, absence of upstream shed vorticity, and smallness of the lateral swimming motions. In sections $2-4$, the hydrodynamic pressure force is analysed, including the local lateral force $\mathscr{L}(x, t)$ and the time-averaged total thrust force $\bar{T}$. In sections 5-6, we illustrate these results by developing a quantitative analysis of the steady-state porpoise bow-riding problem.

\section{The Hydrodynamic Pressure Force}

Cartesian co-ordinates $(x, y, z)$ are employed, fixed with respect to the mean position of the fish, which is facing in the negative $x$-direction with its body axis coincident with the segment $(0, l)$ of the $x$-axis in the "stretchedstraight" position. The body length $l$ is assumed to be $0(1)$, whereas the body thickness is small of order $\varepsilon$, and for simplicity it will be assumed that the body is symmetrically disposed about the $y$ and $z$-axes. In addition the body may perform small lateral motions of its centerplane, normal to the $z$-axis, with displacement $z=h(x, t)$. It is assumed that the flow past the body is inviscid and irrotational, except for thin vortex sheets which are shed only from abrupt trailing edges at the body tail, these tail fins being in the plane $z=0$ when $h=0$.

The "incident" flow experienced by the fish is defined as the velocity field 
which would exist in the absence of the fish. This flow is presumed to be non-uniform, but may be described by a velocity potential $\phi(x, y, z, t)$ which is assumed to vary slowly over distances of order $\varepsilon$, i.e., the lateral dimensions of the fish. Under this assumption, the incident velocity components, in the inner region near the body, can be approximated by Taylor series expansions of the form:

$$
\begin{aligned}
& \phi_{x}=\phi_{x}(x, 0,0, t)+y \phi_{x y}(x, 0,0, t)+z \phi_{x z}(x, 0,0, t)+0\left(\varepsilon^{2}\right), \\
& \phi_{y}=\phi_{y}(x, 0,0, t)+y \phi_{y y}(x, 0,0, t)+z \phi_{y z}(x, 0,0, t)+0\left(\varepsilon^{2}\right), \\
& \phi_{z}=\phi_{z}(x, 0,0, t)+y \phi_{y z}(x, 0,0, t)+z \phi_{z z}(x, 0,0, t)+0\left(\varepsilon^{2}\right) .
\end{aligned}
$$

From the assumption of body symmetry about the $y$ and $z$-axes, it is anticipated that there will be no interactions between the two incident cross-flow velocities $\phi_{y}$ and $\phi_{z}$, even in treating quadratic functionals of the potential such as the longitudinal thrust force acting on the fish. This assumption can be readily confirmed by straightforward extension of the formulae below, and to avoid unnecessary algebra we assume hereafter that the incident field is independent of $y$, or confined to the same plane as the swimming motions of the fish. Then, with the definitions

$$
\begin{aligned}
& U(x, t)=\phi_{x}(x, 0,0, t), \\
& W(x, t)=\phi_{z}(x, 0,0, t),
\end{aligned}
$$

for the two components of the incident velocity on the body axis, it follows from (1) that

$$
\begin{aligned}
& \phi_{x}(x, y, z, t)=U+z W_{x}+0\left(\varepsilon^{2}\right), \\
& \phi_{z}(x, y, z, t)=W-z U_{x}+0\left(\varepsilon^{2}\right),
\end{aligned}
$$

where Laplace's equation has been used in the last equation.

In order to represent the complete flow, including the interaction of the fish body with the incident flow, we write for the total potential,

$$
\Phi=\phi+\psi,
$$

whence $\psi$ represents the disturbance due to the presence of the body, including the effects of its own lateral motion and the disturbance which it causes to the incident flow as well. Appropriate boundary conditions on $\psi$ are that the total normal velocity $\Phi_{n}$ on the body be equal to the normal velocity of the body, and that $\nabla \psi$ vanish at large radial distances from the body axis. In addition, a weak Kutta condition must be imposed at the fish tail. From the slenderness assumption, $\psi$ will satisfy the two-dimensional Laplace equation $\psi_{y y}+\psi_{z z}=0$ in the inner region near the body, with the error a factor $1+O\left(\varepsilon^{2}\right)$, and hence, this potential function can be expanded in the form 


$$
\psi=a_{0} \log r+\sum_{1}^{\infty} a_{m} r^{-m} \cos m \theta+f(x, t)
$$

(cf. Ward, [11], Chapter 9). Here $(r, \theta)$ are polar coordinates,

$$
\begin{aligned}
& y=r \sin \theta, \\
& z-r \cos \theta .
\end{aligned}
$$

The coefficients $a_{m}(x, t)$ are determined from the boundary condition on the body and $f(x, t)$ is related to $a_{0}$ by a linear integral transform. It is unnecessary to solve for all of these coefficients, since the normal velocity on the body is $O(1)$, whereas

$$
(\partial / \partial n) r^{-m}=O\left(\varepsilon^{-m-1}\right)
$$

and hence,

$$
a_{m}=O\left(\varepsilon^{m+1}\right) \text {. }
$$

In fact, the symmetric portion of $\psi$, due to the body thickness, will be one order higher, and hence,

$$
\left(a_{0}, f\right)=O\left(\varepsilon^{2}\right),
$$

as is well known in classical slender-body theory, and

$$
a_{2 m}=O\left(\varepsilon^{2 m+2}\right) \text {. }
$$

Now it is clear that in the "intermediate" region, $\varepsilon \ll r \ll 1$,

$$
\psi \cong a_{0} \log r+\frac{a_{1} \cos \theta}{r}+f+O\left(\frac{\varepsilon^{2 m+2}}{r^{2 m}}, \frac{\varepsilon^{2 m+2}}{r^{2 m+1}}\right), \quad m \geqq 1,
$$

and hence the body's hydrodynamic characteristics are dominated by the source strength $a_{0}$ and dipole moment $a_{1}$. The source strength $a_{0}$ is found from a conservation-of-mass argument, simply by noting that since the body cross-sectional area $S(x)$ is independent of time, the integral of $\Phi_{n}$ taken around the body contour $\Sigma_{B}$ at the station $x=$ constant must vanish, and hence

$$
\int_{\Sigma_{B}}\left(\psi_{n}+\phi_{n}\right) d l=0
$$

the normal $\boldsymbol{n}$ being defined as positive when pointed into the body or out of the fluid domain. Using $(4,5)$ for $\phi_{n}=\phi_{x} n_{x}+\phi_{z} n_{z}$, and (7) to compute the flux due to $\psi_{n}$, it follows that

$$
\begin{aligned}
a_{0} & =\frac{1}{2 \pi} \int_{\Sigma_{B}}\left[\left(U+z W_{x}\right) n_{x}+\left(W-z U_{x}\right) n_{z}\right] d l, \\
& =\frac{1}{2 \pi}\left[U S_{x}+S U_{x}\right]=\frac{1}{2 \pi}(U S)^{\prime},
\end{aligned}
$$

where a prime denotes the partial derivative with respect to $x$. 
The dipole moment $a_{1}(x, t)$ is proportional to the relative lateral velocity $(V-W)$, where

$$
V=h_{\mathrm{t}}+U h_{x} \equiv D h
$$

is the lateral velocity of the body with respect to a (local) moving reference frame translating with velocity $U(x, t)$. This follows by noting that, if the body surface is defined by

$$
F(x, y, z, t)=0=z-h(x, t) \pm g(x, y) \equiv F_{0}-h(x, t),
$$

the boundary condition $0=F_{t}+\nabla \Phi \cdot \nabla F$ takes the form

$$
0=-h_{t}-\Phi_{x} h_{x}+\nabla \Phi \cdot \nabla F_{0},
$$

and the even and odd (in $z$ ) parts of the potential then satisfy the boundary conditions

$$
\begin{aligned}
& \psi_{n}^{\text {(even) }}=-U n_{x}, \\
& \psi_{n}^{\text {(odd) }}=\left(h_{t}+U h_{x}-W\right) n_{z}=(V-W) n_{z},
\end{aligned}
$$

respectively. Then, by a well known theorem (cf. Batchelor, [1], p. 403),

$$
a_{1}(x, t)=-\frac{1}{2 \pi}[A(x)+S(x)](V-W),
$$

where $\rho A(x)$ is the added-mass coefficient of the body contour $\Sigma_{B}$ for lateral acceleration parallel to the $z$-axis.

Having determined the body potential $\psi$ in the intermediate region, in terms of the parameters $S$ and $A$, we may now compute the hydrodynamic pressure forces by utilizing conservation arguments, replacing the direct pressure integral over the body surface (where the full potential $\psi$ is unknown) by a surface integration in the intermediate region. For this purpose, following Newman [6], the vector force $\boldsymbol{F}$ acting on the portion of the fish body upstream of a station $x=$ constant can be expressed in the form

$$
\begin{aligned}
\boldsymbol{F} & =-\rho \iint_{S_{B}}\left[\Phi_{t}+\frac{1}{2}(\nabla \Phi)^{2}\right] n d S \\
& =\rho \iint_{S_{C}}\left[\frac{1}{2}(\nabla \Phi)^{2} n-\Phi_{n} \nabla \Phi\right] d S-\frac{\partial}{\partial t} \iint_{S_{B}} \Phi n d S-\rho i h_{t} \oint_{\Sigma_{B}} \Phi n_{z} d l
\end{aligned}
$$

where $S_{B}$ is the body surface upstream of $\Sigma_{B}$, the intersection of $S_{B}$ and the plane $x=$ constant, and $S_{C}$ is an arbitrary control surface surrounding the partial body surface $S_{B}$ in the fluid.*

* Note that in Newman [6], a (constant) term $-\frac{1}{2} U^{2}$ is introduced in Bernoulli's equation and the subsequent formulae to ensure that the pressure vanishes at infinity; here $U$ is not a constant so that this step must be avoided, but the Bernoulli constant in (12) may be deleted so long as we consider the longitudinal force $F_{x}$ only for the complete closed body, where $\iint \boldsymbol{n} d S=0$, and the local lateral force $\mathscr{L}(x, t)$ for a closed body section where $\oint n_{y} d l=0$. 
We shall use (12) to find the local lateral force $\mathscr{L}(x, t)=(\partial / \partial x) F_{z}$, and the total longitudinal drag (or thrust) force $F_{x}$. In both cases, the control surface $S_{\mathrm{C}}$ is taken to be (Fig. 1):

(1) a circular cylinder $r=$ constant in the intermediate region outboard of the body, denoted by $S_{I}$;

(2) a ciusing suifface $S_{\infty}$ far upstream, on which $\psi=0$; and

(3) a closing surface $S_{T}$ between $S_{I}$ and $S_{B}$ in the plane $x=$ constant, outboard of $\Sigma_{B}$.

It will be convenient to introduce a "base area" surface $S_{0}$ interior to the body in the same plane as $S_{T}$; thus $S_{0}$ is the plane surface interior to and bounded by $\Sigma_{B}$.

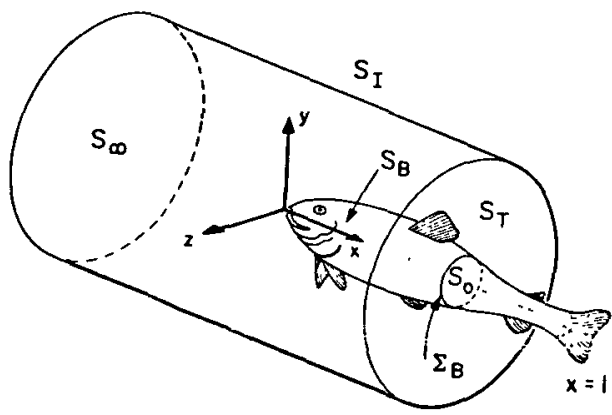

Figure 1.- Coordinate system and control surface surrounding the upstream portion of the body surface.

\section{Local Lateral Force}

The lateral force component $F_{z}$, acting upon the portion of the body upstream of $S_{T}$, is given by

$$
\begin{aligned}
& F_{z}=\rho \iint_{S_{x}} \phi_{x} \phi_{z} d y d z+\rho \iint_{S_{1}}\left[\frac{1}{2}\left(\phi_{x}^{2}+\phi_{z}^{2}\right) n_{z}-\phi_{n} \phi_{z}\right] d S-\rho \iint_{S_{\mathbf{r}}} \phi_{x} \phi_{z} d y d z \\
& +\rho \iint_{S_{1}}\left[\left(\phi_{x} \psi_{x}+\phi_{z} \psi_{z}\right) n_{z}-\phi_{x} \psi_{z}-\psi_{n} \phi_{z}\right] d S-\rho \iint_{S_{\mathbf{T}}}\left(\phi_{x} \psi_{z}+\psi_{x} \phi_{z}\right) d y d z \\
& +\rho \iint_{S_{1}}\left[\frac{1}{2}\left(\psi_{x}^{2}+\psi_{y}^{2}+\psi_{z}^{2}\right) n_{z}-\psi_{n} \psi_{z}\right] d S-\rho \iint_{S_{T}} \psi_{x} \psi_{z} d y d z \\
& -\rho \frac{\partial}{\partial t} \iint_{S_{8}}(\phi+\psi) n_{z} d S
\end{aligned}
$$

The first three integrals, involving quadratic products of derivatives of $\phi$, can be evaluated simply by noting that 


$$
\rho \iint_{S_{\infty}+S_{S_{1}+S_{T}}}\left[\frac{1}{2}(\nabla \phi)^{2} n-\phi_{n} \nabla \phi\right] d S=-\rho \iint_{S_{0}}\left[\frac{1}{2}(\nabla \phi)^{2} n-\phi_{n} \nabla \phi\right] d S
$$

since $\phi$ is harmonic in the interior of the closed surface $S_{\infty}+S_{1}+S_{T}+S_{0}$. Using (4-5) the right side of this equation can be evaluated, and the $z$ component is simply

$$
\rho \iint_{S_{0}} \phi_{x} \phi_{z} d y d z=\rho U W S+O\left(\varepsilon^{3}\right) .
$$

The remaining integrals over $S_{l}$ are evaluated using (4-5) for $\left(\phi_{x}, \phi_{z}\right)$ and (8) for $\psi$, and consistently neglecting terms of $O\left(\varepsilon^{2} r\right)$ or higher in $r$. It then-follows that

$$
\begin{aligned}
& \rho \iint_{S_{I}}\left[\left(\phi_{x} \psi_{x}+\phi_{z} \psi_{z}\right) n_{z}-\phi_{n} \psi_{z}-\psi_{n} \phi_{z}\right] d S \\
& \quad=\pi \rho U(x) a_{1}(x, t)-2 \pi \rho \int_{0}^{x} d x\left[a_{0}(x, t) W(x, t)+a_{1}(x, t) U^{\prime}(x, t)\right],
\end{aligned}
$$

and the comparable integral involving $\psi^{2}$ vanishes to $O\left(\varepsilon^{2}\right)$. For the integrals over $S_{T}$, the leading-order term is

$$
-\rho \iint_{S_{T}} \phi_{x} \psi_{z} d y d z \cong-\rho U \iint_{S_{\tau}} \psi_{z} d y d z=-\rho U \oint_{\Sigma_{B}} \psi n_{z} d l-\pi \rho U(x) a_{1}(x, t) .
$$

Finally, the integral over the body surface $S_{B}$ is

$$
\begin{aligned}
-\rho \frac{\partial}{\partial t} \iint_{S_{B}}(\phi+\psi) n_{z} d S & =\rho \frac{\partial}{\partial t} \iiint \phi_{z} d t-\rho \frac{\partial}{\partial t} \int_{0}^{x} \oint_{\Sigma_{B}} \psi n_{z} d l \\
& =\rho \int_{0}^{x} W_{I} S d x-\rho \frac{\partial}{\partial t} \int_{0}^{x} \oint_{\Sigma_{B}} \psi n_{z} d l .
\end{aligned}
$$

Adding these results together,

$$
\begin{aligned}
F_{z}= & -2 \pi \rho \int_{0}^{x} d x\left(a_{0} W+a_{1} U^{\prime}\right)+\rho \int_{0}^{x} d x S W_{t}+\rho U W S-\rho U \oint_{\Sigma_{B}} \psi n_{z} d l \\
& -\rho \frac{\partial}{\partial t} \int_{0}^{x} d x \oint_{\Sigma_{B}} \psi n_{z} d l \equiv \int_{0}^{x} \mathscr{L}(x, t) d x,
\end{aligned}
$$

where

$$
\begin{aligned}
\mathscr{L}(x, t)= & -2 \pi \rho\left(a_{0} W \cdot+a_{1} U^{\prime}\right)+\rho D(W S)-\rho D\left(\oint_{\Sigma_{B}} \psi n_{z} d l\right) \\
& +\rho U^{\prime} W S-\rho U^{\prime}\left(\oint_{\Sigma_{B}} \psi n_{z} d l\right) .
\end{aligned}
$$


Finally, if we note the relations $(9,11)$ for $a_{0}$ and $a_{1}$, and the relation (cf. Newman and $\mathrm{Wu},[9]$, section 6),

$$
\int_{\Sigma_{B}} \psi n_{z} d l=A(V-W),
$$

equation (17) reduces to

$$
\mathscr{L}(x, t)=-\rho D[A(V-W)]+\rho S D(W)+\rho U^{\prime} S(V-W) .
$$

Equation (19) is the desired expression for the differential lateral force acting on the body section, as a result of the combined effects of the lateral body velocity $V(x, t)$ and the non-uniform inflow velocity field $[U(x, t)$, $W(x, t)]$. Special cases of this equation have been derived by other authors in a variety of contexts. In his original treatment of slender-body theory, Munk (cf. Durand, 1963, Vol. 6, pp. 41-45) treated the case of steady-state motion with constant $U, V$ and $W=0$, for an axi-symmetric body. Nielsen et al [9] generalize Munk's formula to the case of a non-uniform but steady lateral inflow $W(x)$, and axi-symmetric body. Lighthill [5] analyses the unsteady swimming motion of slender fish in a uniform field, corresponding to the special case of (19) where $U=$ constant and $W=0$. Tuck and Newman [10] present a heuristic derivation of (19) for $U=$ constant, in order to predict the interaction forces between adjacent ships.

The first term in (19) can be interpreted as the inertial force due to the resultant velocity $(V-W)$ of the body relative to the fluid, this motion imparting a momentum $\rho A(V-W)$ to the fluid and a change of momentum equal to $D[\rho A(V-W)]$. The second term represents the effects of the inhomogeneous incident velocity field pressure gradient, which exerts an effective bouyancy force on the cross-section proportional to the cross-section area $S$, and the last term is an interaction effect between the lateral motion and the longitudinal velocity gradient.

\section{Total Thrust Force}

The total longitudinal force $F_{x}$ may be computed in a similar fashion from (12), taking the plane $S_{T}$ and contour $\Sigma_{B}$ at the body tail $x=l$. In this case there is no contribution from terms quadratic in $\phi$, and

$$
\begin{aligned}
F_{x}= & -\rho \iint_{S_{1}}\left(\phi_{n} \psi_{x}+\psi_{n} \phi_{x}+\psi_{n} \psi_{x}\right) d S-\rho \iint_{S_{T}}\left(\phi_{x} \psi_{x}-\phi_{z} \psi_{z}\right. \\
& +\frac{1}{2} \psi_{x}^{2}-\frac{1}{2} \psi_{y}^{2}-\frac{1}{2}\left[\psi_{z}^{2}\right) d y d z \\
& -\rho h_{t} \oint_{\Sigma_{B}} \psi n_{z} d l-\rho \frac{\partial}{\partial t} \iint_{S_{B}} \Phi n_{x} d S .
\end{aligned}
$$


Hereafter, we consider only the time-average $\bar{F}_{x}$, whence the last term in (20) drops out if the motion is periodic or steady-state. Substituting for $\phi$ and $\psi$ on $S_{l}$ then gives

$$
\begin{aligned}
-\rho \iint_{S_{1}}\left(\phi_{n} \psi_{x}+\psi_{n} \phi_{x}+\psi_{n} \psi_{x}\right) d S= & -2 \pi \rho \int_{0}^{l}\left(U a_{0}-W^{\prime} a_{1}\right) d x \\
& -\pi \rho W(l, t) a_{1}(l, t) \\
& +O\left(\varepsilon^{2} r^{2} \log r\right) .
\end{aligned}
$$

Similarly, for the integral over $S_{\boldsymbol{T}}$, the leading-order terms are:

$$
\begin{aligned}
\rho \iint_{S_{T}}\left[\phi_{z} \psi_{z}\right. & \left.+\frac{1}{2}\left(\psi_{y}^{2}+\psi_{z}^{2}\right)\right] d y d z \\
& =\rho W \oint_{\Sigma_{B}+\Sigma_{I}} \psi n_{z} d l+\frac{1}{2} \rho \oint_{\Sigma_{B}} \psi \psi_{n} d l \\
& =\pi \rho\left(W a_{1}\right)_{x=1}+\frac{1}{2} \rho \int_{\Sigma_{B}} \psi\left[2 W n_{z}+\psi_{n}\right] d l .
\end{aligned}
$$

where $\Sigma_{I}$ denotes the intersection of $S_{T}$ and $S_{l}$. Adding these contributions, the time-average of $(20)$ gives

$$
\bar{F}_{x}=2 \pi \rho \int_{0}^{l}\left(-U a_{0}+W^{\prime} a_{1}\right) d x+\frac{1}{2} \rho \oint_{\Sigma_{B}} \psi\left[\left(2 W-2 h_{t}\right) n_{z}+\psi_{n}\right] d l .
$$

Substituting for $a_{0}$ and $a_{1}$ from $(9,11)$, and applying the boundary condition for $\psi_{n}$ on $\Sigma_{B}$,

$$
\bar{F}_{x}=\rho \int_{0}^{l}\left[-\overline{U(U S)^{\prime}}+\overline{W^{\prime}(W-V)}(A+S)\right] d x+\frac{1}{2} \rho \oint_{\Sigma_{B}} \overline{\psi\left[W-h_{t}+U h_{x}\right]} n_{z} d l .
$$

Finally, since the trailing edge is planar, the jump in the potential $[\psi]$ must be

$$
[\psi]=2(W-V)\left(y^{2}-s^{2}\right)^{1 / 2},
$$

where $s$ is the semi-span at $x=l$ (cf. Newman, [6], eq. 19). Substituting in (22) then yields

$$
\bar{F}_{x}=\rho \int_{0}^{1}\left[-\overline{U(U S)^{\prime}}+\overline{W^{\prime}(W-V)}(A+S)\right] d x+\frac{1}{2} \pi \rho s^{2}\left(\overline{V-W)\left(W-h_{\mathrm{t}}+U h_{x}\right.}\right)
$$

Noting that $A(l)=\pi s^{2}$, and integrating by parts, the thrust $\bar{T}=-\bar{F}_{x}$ is

$$
\bar{T}=\rho \int_{0}^{l}\left[-\overline{U U^{\prime} S}+\overline{W^{\prime}(V-W}(A+S)\right] d x+\frac{1}{2} \rho A(l)\left[\left(\overline{\left.h_{t}-W\right)^{2}}-\overline{U^{2} h_{x}^{2}}\right]_{x=I} .\right.
$$


Equation (23) is the desired expression for the average thrust $\bar{T}$. For a uniform inflow $W=0, U=$ constant, this reduces to the expression originally derived by Lighthill [5].

The component in (23) proportional to $A(l)$ is a straightforward generalization of Lighthill's [5] formula to account for the change of inflow vector from $(U, 0)$ to $(U, W)$. The integral over the body represents the inhomegeneous effects, the term $U U^{\prime} S$ being the bouyancy force due to the pressure gradient of the axial velocity component. The second term in the integral is due to the longitudinal pressure gradient associated with the lateral flow interaction, and may be compared with various explanations of porpoise bow-riding in the presence of a curved flow or "suddenly-changing flow direction".

\section{Applications to Steady-state Porpoise Bow-riding}

For a porpoise, in steady motion near an adjacent ship's bow, the unsteady terms in (19) and (23) vanish, leaving

$$
\begin{gathered}
\mathscr{L}(x)=-\rho U[A(V-W)]^{\prime}+\rho U S W^{\prime}+\rho U^{\prime} S(V-W), \\
\bar{T}=\rho \int_{0}^{l}\left(-U U^{\prime} S+W^{\prime}(V-W)(A+S)\right] d x+\frac{1}{2} \rho A(l)\left[W^{2}-V^{2}\right]_{x=1},
\end{gathered}
$$

and

$$
V=U h_{x} .
$$

We now inquire under what circumstances the porpoise will be in equilibrium, that is,

$$
\begin{gathered}
\int_{0}^{1} \mathscr{L}(x) d x=0, \\
\int_{0}^{1} \mathscr{L}(x) x d x=0, \\
\bar{T}=D=(\text { viscous }) \text { drag force, }
\end{gathered}
$$

where the first two equations express equilibrium of the lateral force and yaw moment.

The simplest case to consider is that of a purely-longitudinal incident flow, with $W=0$, as at the bow stagnation point and forward of this point on the centerplane of a ship. Then, with $V=0$, the equilibrium condition is simply

$$
\bar{T}=-\rho \int_{0}^{1} U U^{\prime} S d x=D .
$$

As an example, we set $D=\frac{1}{2} \rho U^{2} D^{\prime}$ where $D$ ' is the "drag area" which has been experimentally measured by Lang [4]. Assuming, in addition, that 
$I U^{\prime} / U \ll 1$, or the porpoise length is short compared to the scale of the velocity gradient, (29) can be integrated to give

$$
\frac{1}{2} \rho U^{2} D^{\prime}=-\rho U U^{\prime} \int_{0}^{l} S d x=-\rho U U^{\prime} \forall,
$$

or

$$
U^{\prime} / U=-\frac{1}{2} D^{\prime} / \forall,
$$

where $\forall$ is the displaced volume of the porpoise. Typical data from Lang [4] are for a porpoise with $\forall=0.1 \mathrm{~m}^{3}$, and show $D^{\prime}=0.06 \pm 0.002 \mathrm{~m}^{2}$. Thus this porpoise will be in equilibrium if

$$
U^{\prime} / U=-0.03 m^{-1} \text {. }
$$

which is typical of the velocity gradients $2-5 \mathrm{~m}$. forward of the bow on typical ships.

Next, we consider the possibility of equilibrium in a "suddenly-changing flow direction", $W^{\prime} \neq 0$, but with (locally) uniform longitudinal flow, $U=$ constant. This requires that

$$
\begin{gathered}
0=\int_{0}^{1} \mathscr{L}(x) d x=-\rho U[A(V-W)]_{x=1}+\rho U \int_{0}^{1} S W^{\prime} d x \\
0=\int_{0}^{1} x \mathscr{L}(x) d x=-\rho U l[A(V-W)]_{x-1}+\rho U \int_{0}^{1}\left[x S W^{\prime}+A(V-W)\right] d x
\end{gathered}
$$

and

$$
\bar{T}=\rho \int_{0}^{1} W^{\prime}(V-W)(A+S) d x+\frac{1}{2} \rho\left[A\left(W^{2}-V^{2}\right)\right]_{x=1}>0 .
$$

We assume without loss of generality that $W^{\prime}>0$. Then, from (33), $(V-W)>$ 0 at the tail $x=l$. Subracting $l$ times (33) from (34),

$$
0=\rho U \int_{0}^{l}\left[(x-l) S W^{\prime}+A(V-W)\right] d x,
$$

and therefore,

$$
\int_{0}^{1} A(V-W) d x=\int_{0}^{l}(l-x) S W^{\prime} d x>0 .
$$

Thus, for lateral force and moment equilibrium, $A(V-W)$ should be an increasing function of $x$, with maximum value

$$
\int_{0}^{1} S W^{\prime} d x>0
$$


at the tail, and mean value

$$
\int_{0}^{l}(l-x) S W^{\prime} d x>0,
$$

along the body. A sufficient condition that (35) be satisfied is that $V+W<0$, and a necessary condition for positive thrust, without large values of $(V-W)$ which lead to separation, is that $W<V<0$. Thus the assumed configuration is as shown in Fig. 2, along with the "mirror" solution $W^{\prime}<0, W>V>0$. Only the latter case is relevant to bow-riding (except on the back side of the bow-wave!) and thus we may conclude that the thrust force resulting from the longitudinal pressure gradient may be enhanced by the vertical velocity field if $W>0$ but $W^{\prime}<0$.
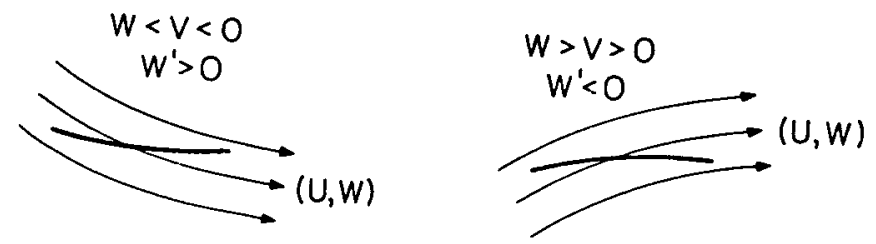

Figure 2.- Configurations of body axis (heavy line) so as to generate a positive (to the left) thrust in the presence of the curved streamlines shown by the light lines.

\section{Porpoise Wave-riding}

Finally, we consider the possibility of a porpoise riding on an oblique wave alongside a ship or on an ambient surface wave. In this case the incident fields is

$$
\phi=\frac{g H}{2 \omega} e^{k z+i k(x \cos \theta+y \sin \theta)}+U_{0} x,
$$

where $H$ is the wave height (twice the wave amplitude), $g$ is the gravitational acceleration, $k$ the wave number, and $\omega=\sqrt{g h}$ the wave frequency in a fixed reference system. The vertical coordinate $z$ is positive upward, deep water is assumed, and the real part is to be taken in all complex expressions. The wave angle $\theta$ is the angle between the direction of wave propagation and the negative $\boldsymbol{x}$-axis, and the wave will be steady in the moving reference frame, moving to the left with velocity $U_{0}$, if $k=g U_{0}^{-2} \sec ^{2} \theta$. Now the porpoise must accomodate itself to the three-dimensional field 


$$
\begin{aligned}
& \phi_{x}=U_{0}+\frac{1}{2} i H(g k)^{1 / 2} \cos \theta e^{k z_{0}+i k x \cos \theta}, \\
& \left.\begin{array}{c}
\phi_{y} \\
\phi_{z}
\end{array}\right\}=\frac{1}{2}\left\{\begin{array}{c}
i \sin \theta \\
1
\end{array}\right\} H(g k)^{1 / 2} e^{k z_{0}+i k x \cos \theta},
\end{aligned}
$$

on the $x$-axis at depth $z_{0}<0$ below the free surface.

If the wave height is small (which is necessary for the above expression to hold) the only first-order contribution to the thrust (25) is from the longitudinal pressure gradient, or

$$
\bar{T}=-\rho U_{0} \int_{0}^{1} \phi_{x x} S d x+O\left(H^{2}\right),
$$

and if the porpoise length $l$ is small compared to wavelength $2 \pi / k$,

$$
\begin{aligned}
\bar{T} & \cong-\rho U_{0} \forall \phi_{x x} \\
& =\frac{1}{2} \rho U_{0} \forall k \dot{H}(g k)^{1 / 2} \cos ^{2} \theta e^{k z_{0}+i k x \cos \theta} \\
& =\frac{1}{2} \rho g \forall k H e^{k z_{0}+i k x \cos \theta} \cos \theta .
\end{aligned}
$$

Noting that the wave elevation is in phase with the oscillatory component of $\phi_{x}$, it follows that $\bar{T}$ is a maximum on the wave face, at the wave node $x=0$ where the slope is a maximum. Before considering the rather complex question of force and moment equilibrium, we note that the maximum thrust will overcome the drag reported by Lang [4] if

$$
\frac{1}{2} \rho g \forall k H e^{k z_{0}} \cos \theta=\frac{1}{2} \rho U_{o}^{2} D^{\prime},
$$

where $D^{\prime}=0.06 \mathrm{~m}^{2}, \forall=0.1 \mathrm{~m}^{3}$. and thus

$$
\frac{1}{2} k H e^{k z_{0}} \cos \theta=0.03 U_{0}^{2} / g .
$$

The left side of this expression is the $x$-component of the streamline slope at depth $z_{0}$. If $U_{0}=6 \mathrm{~ms}^{-1}$, corresponding to a ship speed of 12 knots, it follows that the local wave slope must be .12 radians or 7 degrees. The generally accepted maximum wave slope on the surface for a permanent wave system is $30^{\circ}$; this will be reduced at depths $z_{0}<0$, but also may be exceeded locally in a ship's bow wave system.

In addition it is necessary to consider the lateral force and moment equilibrium for the porpoise in the three-dimensional wave field. For this 
purpose we assume the porpoise to be rotated through some arbitrary angle $\chi$ relative to the vertical, and note that the tail fins are inclined through the same angle to the horizontal (See Fig. 3 ). In body-fixed coordinates $\left(y_{p}, z_{p}\right)$ the local force (24) is valid separately for the $y_{p}$ and $z_{p}$ components of the lateral force, the only difficulty being to discriminate the appropriate values of added mass $A$ and velocities $(V, W)$. For simplicity we may assume that $A=S$ for porpoise motions along the $y_{p}$-axis (an exact result if the porpoise body is axisymmetric, with only a tail fin appendage) but, as a result of the tail fins, $A \neq S$ for $z_{p}$-modes. Then, from (24),

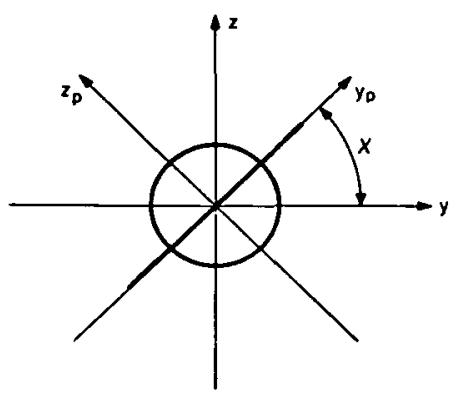

Figure 3.- Rotated coordinate system $\left(y_{p}, z_{p}\right)$ fixed to the porpoise body and tail (heavy lines).

$$
\mathscr{L}_{y p}=-\rho U_{0}\left\{\left[S\left(V_{y p}-W_{y p}\right)\right]^{\prime}+S W_{y p}^{\prime}\right\},
$$

where the second-order term involving products of $U^{\prime}$ and $V, W$ is neglected. Similarly,

$$
\mathscr{L}_{z p}=-\rho U_{0}\left\{\left[A_{z p}\left(V_{z p}-W_{z p}\right)\right]^{\prime}+S W_{z p}{ }^{\prime}\right\} .
$$

By vector summation,

and thus

$$
\begin{aligned}
& W_{y p}=\phi_{y} \cos \chi+\phi_{z} \sin \chi, \\
& W_{z p}=\phi_{z} \cos \chi-\phi_{y} \sin \chi,
\end{aligned}
$$

$$
\left\{\begin{array}{l}
W_{y p} \\
W_{z p}
\end{array}\right\}=\frac{1}{2} H(g k)^{1 / 2} e^{k z_{0}+i k x \cos \theta}\left\{\begin{array}{l}
i \sin \theta \cos \chi+\sin \chi \\
\cos \chi-i \sin \theta \sin \chi
\end{array}\right\}
$$


The two lateral force equilibrium conditions are

$$
\int_{0}^{\prime} \mathscr{L} d x=\left\{\begin{array}{c}
\int S W_{y p}^{\prime} d x \\
{\left[A_{z p}\left(V_{z p}-W_{z p}\right)\right]_{x=1}+\int S W_{z p}^{\prime}}
\end{array}\right\}=0,
$$

and the corresponding moment equations are

$$
\int_{0}^{l} \mathscr{L} x d x=\left\{\begin{array}{c}
\int x S W_{y p}{ }^{\prime} d x-\int S\left(V_{y p}-W_{y p}\right) d x \\
\int x S W_{z p}{ }^{\prime}+l\left[A_{z p}\left(V_{z p}-W_{z p}\right)\right]_{x=1}-\int A_{z p}\left(V_{z p}-W_{z p}\right) d x
\end{array}\right\}=0 .
$$

The first of these four equations requires that

$$
\operatorname{Re}\{i k \cos \theta[i \sin \theta \cos \chi+\sin \chi]\}=0
$$

(to leading order in $k l \ll 1$, since $x=0$ is the position of maximum thrust). Thus, for $\theta>0, \chi=90^{\circ}$ and the porpoise must swim on its side. (Note this could be anticipated, since $\phi_{x y}$ is a (negative) maximum and $\phi_{x z}=0$ on the face.] Now, with $\chi=90^{\circ}$ (and hence $W_{y p}{ }^{\prime}=W_{z p}=0$ at $x=0$ ) equilibrium of the $z_{p}$-force requires that

$$
\begin{aligned}
{\left[A_{z p} V_{z p}\right]_{x=1} } & =-\int_{0}^{1} S W_{z p}^{\prime} d x \\
& \cong-\frac{1}{2} k H(g k)^{1 / 2} \sin \theta \cos \theta \forall e^{k z_{0}}
\end{aligned}
$$

which prescribes the value of $V_{z p}=U_{0} h_{x}$ at the tail. Equilibrium of the two moments requires that

$$
\begin{aligned}
\int_{0}^{1} S V_{y p} d x & =\int_{0}^{1} S W_{y p} d x \\
& \cong \frac{1}{2} \forall H(g k)^{1 / 2} e^{k z_{0}},
\end{aligned}
$$

and

$$
\begin{aligned}
\int_{0}^{1} A V_{z p} d x & =\int_{0}^{l} x S W_{z p}{ }^{\prime}+l\left[A_{z p} V_{z p}\right]_{x=1} \\
& \cong-\frac{1}{2} k H \forall(g h)^{1 / 2} \sin \theta \cos \theta e^{k z_{0}}\left(l-x_{c}\right),
\end{aligned}
$$


where $x_{c}$ is the centroid position of the porpoise:

$$
x_{c}=\frac{1}{\forall} \int_{0}^{1} x S(x) d x .
$$

These two moment equations may be satisfied by suitable choice of $V_{y p}>0$ along the body length. Hence we conclude that bow-riding alongside the ship's bow on an oblique bow wave is indeed possible, provided the porpoise swims on its side. Observations of porpoises riding oblique waves in this manner are not known to the author, but Fejer and Backus [3] report that porpoises do swim on their side adjacent to ships' bows off to one side of the centerplane; presumably in this case the longitudinal pressure gradient is the principal thrust-producing mechanism, but the lateral deflection of streamlines around the vertical bow requires that the porpoise swim on its side to obtain equilibrium of the lateral force and vertical yaw moment.

\section{Acknowledgement}

This work was initiated during a visit to the University of Adelaide, with support from the Australian-American Education Foundation and the John Simon Guggenheim Memorial Foundation, and was completed with support from the National Science Foundation, Grant GK-43886X, and the Office of Naval Research, Contract N00014-67-A-0204-0023.

\section{References}

[1] G. K. Batchelor, An Introduction to Fluid Mechanics, Cambridge University Press, (1967).

[2] R. Coene, The Swimming of Slender Fish-like Bodies in Waves, Delft University of Technology, The Netherlands, (1974).

[3] A. A. Fejer and R. H. Backus, 'Porpoises and the Bow-riding of Ships Underway', Nature 188 (1960), 4752, 700-703.

[4] T. G. Lang, 'Hydrodynamic Analysis of Cetacean Performance', Whales, Dolphins and Porpoises, K. S. Norris, Ed., 410-432, University of California Press, Berkeley, (1966).

[5] M. J. Lighthill, 'Note on the Swimming of Slender Fish', Journal of Fluid Mechanics 4 (1960), $397-430$.

[6] J. N. Newman, 'The Force on a Slender Fish-like Body', Journal of Fluid Mechanics 58 (1973), Part 4, 689-702.

[7] J. N. Newman and T. Y. Wu, 'A Generalized Slender-body Theory for Fish-like Forms', Journal of Fluid Mechanics 57 (1973), Part 4, 673-693.

[8] J. N. Newman and T. Y. Wu, 'Hydromechanical Aspects of Fish Swimming', Symposium on Swimming and Flying in Nature, Calif. Inst. of Tech. (1974).

[9] J. N. Nielsen, F. K. Goodwin and F. E. Dillenius, 'A Calculative Method for Predicting Store Separation Trajectories at Speeds up to the Critical Speed', Specialists Meeting on Aerodynamic Performance, AGARD-CP-71-71, paper No. 26, (1970). 
[10] E. O. Tuck and J. N. Newman, 'Hydrodynamic Interactions Between Ships', Tenth Sym posium on Naval Hydrodynamics, Cambridge, Mass., June, 1974 (Proceedings to be published by Office of Naval Research, Washington, D. C.)

[11] G. N. Ward, Linearized Theory of Steady High-Speed Flow, University Press, Cambridge, England, (1955).

[12] D. Weihs, 'Hydromechanics of Fish Schooling', Nature, 241 (1973), 290-291.

Department of Ocean Engineering,

Massachusetts Institute of Technology,

Cambridge, Mass. 02139. 